\title{
Effectiveness and Tolerability of Perampanel in Children and Adolescents with Refractory Epilepsies: First Experiences
}

Anna Biró ${ }^{1} \quad$ Ulrich Stephani ${ }^{2}$ Tiziana Tarallo ${ }^{2}$ Thomas Bast ${ }^{3}$ Kurt Schlachter ${ }^{4}$ Martin Fleger ${ }^{4}$ Gerhard Kurlemann $^{5}$ Barbara Fiedler ${ }^{5}$ Steffen Leiz ${ }^{6}$ Marina Nikanorova ${ }^{7}$ Markus Wolff ${ }^{8}$ Arnd Müller ${ }^{1}$ Christina Selch ${ }^{1}$ Martin Staudt ${ }^{1,9}$ Gerhard Kluger ${ }^{1,10}$

\footnotetext{
${ }^{1}$ Department of Pediatric Neurology and Neurological Rehabilitation, Schön Klinik Vogtareuth, Vogtareuth, Germany

2 Department of Neuropediatrics, Christian-Albrechts University, University Medical Center Schleswig-Holstein, Kiel, Germany

${ }^{3}$ Epilepsy Center Kork, Kehl, Germany

${ }^{4}$ Department of Pediatrics, Bregenz Regional Hospital, Bregenz, Austria

${ }^{5}$ Department of Neuropediatrics, University Children's Hospital Münster, Münster, Germany

${ }^{6}$ Department of Pediatrics, Dritter Orden Clinic, München, Germany

${ }^{7}$ Children's Department, Danish Epilepsy Centre, Dianalund, Denmark

${ }^{8}$ Department of Pediatric Neurology, University Children's Hospital Tübingen, Tübingen, Germany

9 University Children's Hospital Tübingen, Tübingen, Germany

10 Paracelsus Medical University, Salzburg, Austria
}

Address for correspondence Anna Biró, MD, Department of Pediatric Neurology and Neurological Rehabilitation, Epilepsy Center for Children and Adolescents, Schön Klinik Vogtareuth, Krankenhausstraße 20, 83569 Vogtareuth, Germany (e-mail: abiro@schoen-kliniken.de).

Neuropediatrics 2015;46:110-115.

\begin{abstract}
Keywords

- perampanel

- pediatric

- refractory epilepsies

- treatment outcome

- adverse effects

Objective This article aims to report the first clinical experiences concerning effectiveness and tolerability of perampanel (PER) in a pediatric population with refractory epilepsies.

Patients and Methods This nonsponsored, observational, retrospective survey was conducted through collaboration with multiple centers in Europe. The clinical course of the first pediatric patients treated in these centers with PER was documented with the help of a questionnaire completed by the treating physicians. Effectiveness and adverse effects were evaluated. The study population consisted of 58 patients (mean age, 10.5 years; range, 2-17 years), suffering from various refractory epilepsies, classified as focal epilepsy $(n=36)$, unclassified generalized epilepsy $(n=12)$, Lennox-Gastaut syndrome $(n=5)$, West syndrome $(n=3)$, and Dravet syndrome $(n=2)$.

Results The response rate ( $\geq 50 \%$ seizure reduction) after the first 3 months of therapy was $31 \%$ (18/58 patients) in total. Complete seizure control was achieved in five patients ( $9 \%$ overall). Aggravation of seizures occurred in five cases $(9 \%)$. The most frequently occurring adverse effects were reduced vigilance or fatigue $(n=16)$ and behavioral changes $(n=14)$.

Discussion PER seems to be effective also in children and adolescents with pharmacorefractory epilepsies. Tolerability was acceptable.
\end{abstract}

received

September 5, 2014

accepted after revision

January 1, 2015

published online

March 2, 2015 (c) 2015 Georg Thieme Verlag KG

Stuttgart · New York
DOI http://dx.doi.org/ 10.1055/s-0035-1546276. ISSN 0174-304X. 


\section{Introduction}

Perampanel (PER) is an orally active, selective, noncompetitive antagonist of the AMPA glutamate receptor on postsynaptic neurons. $^{1,2}$

Three phase III trials were conducted in adults and adolescents, assessing the clinical efficacy and tolerability of PER, providing class I evidence that adjunctive PER is effective in reducing partial-onset seizures with acceptable tolerability. Adverse events occurred in a dose-dependent manner. ${ }^{3-5}$

The half-life of PER is approximately 105 hours, so that steady state is reached in approximately 2 to 3 weeks. There is a linear relationship between the administered dose and PER plasma concentrations. 6 , With an increased PER plasma concentration, there is an increase in efficacy, but the higher plasma concentrations are also significantly associated with increased risk of adverse events. ${ }^{8}$

A marketing authorization was issued throughout the European Union in July 2012 for the adjunctive treatment of partial-onset seizures with or without secondary generalization in patients with epilepsy aged 12 years and older.

The first reported clinical experiences with adjunctive PER treatment in an adult population after 6 months of therapy with PER were promising99: 34 of 74 (46\%) of the patients were responders with a reduction of seizure frequency by at least $50 \%$.

A European Medicines Agency (EMA) decision was made on a pediatric investigation plan for PER. According to the last modification (June $2014^{10}$ ), the completion of the pediatric investigations is planned by April 2021.

To our knowledge, no studies including children younger than 12 years have been completed on PER treatment to date. Through lack of pediatric data on PER, neuropediatric specialists currently can only extrapolate data from the existing adult studies, while considering the off-label use of the PER for their young epilepsy patients who have proven themselves resistant to the approved antiepileptic drugs (AEDs) and their combinations. This is a well-known issue concerning pediatric drug development and pediatric AED development, yet to be solved. ${ }^{11}$

The purpose of this survey was to collect and share the first clinical experiences with PER in a pediatric population, as further evidence-based data are only to be expected in the far future.

\section{Methods}

We performed a retrospective, noninterventional survey, without sponsoring. Data collection was conducted through collaboration with tertiary epilepsy centers in Germany, Austria, and Denmark (-Supplementary Table 1, onlineonly), invited to participate via personal correspondence. The participating neuropediatricians reported the clinical course of their first pediatric patients started on a PER until April 2013, with the help of a questionnaire. The minimum observation period was 16 weeks after the introduction of PER. Observation is ongoing and planned until an 18 months follow-up.

Seizure types and frequency were determined clinically (documented by parents or medical staff) or by previous examinations (such as video electroencephalography monitoring). Epilepsy syndromes and seizure types were classified according to the International League Against Epilepsy (ILAE) classification. ${ }^{12}$

Initial dosage and titration schedule of PER were at the discretion of the treating physician according to medical need. Effectiveness was evaluated by comparing the frequency of seizures between baseline (the 4 weeks before PER therapy) and another 4-week period, 3 months after initiation, as reported by the caregivers. Responders were defined as patients whose seizure frequency was reduced by at least $50 \%$. Aggravation of seizures was defined as an increase in seizure frequency by at least $50 \%$.

Tolerability was assessed through documentation of possible adverse effects during treatment, rated on a 3-level scale (mild, moderate, and severe) by the treating physician. The acquired data were checked for plausibility and completeness and were entered into a database.

\section{Patients}

We collected the data of 59 children and adolescents, suffering from various therapy refractory epilepsies, who underwent treatment with PER. Of these, one patient had to be excluded because of inconsistent data. Two of the patients have already been reported. ${ }^{9}$

Our population consisted of 58 children and adolescents (32 females). Age ranged from 2 to 17 years (mean, $10.5 \pm 4.2$ years). Age distribution was as follows: 11 children were of 2 to 5 years (one 2-year-old, three 3-year-olds, two 4-year-olds, and five 5-year-olds), 22 children were 6 to 11 years, and 25 children were aged 12 years and older. Mean age at epilepsy onset was $2.6 \pm 2.6$ years (range, $0-9$ years). Mean duration of epilepsy was $8.1 \pm 4.3$ years (range, $1.8-15.8$ years).

Epilepsy etiology was structural-metabolic in 27 patients (46.5\%), unknown in 20 patients (34.5\%), and genetic in 11 patients (19\%). Epilepsy syndromes were classified as focal epilepsy $(n=36)$, unclassified generalized epilepsy $(n=12)$, Lennox-Gastaut syndrome $(n=5)$, West syndrome $(n=3)$, and Dravet syndrome $(n=2)$.

Focal seizures $(n=27)$ and generalized tonic seizures $(n=16)$ were the most frequently reported dominant seizure types. Generalized tonic-clonic seizures were the dominant seizure type of four patients, absences in six patients (four atypical and two myoclonic), generalized myoclonic seizures in two, and epileptic spasms in three patients.

Epilepsies were uncontrolled by sequential monotherapy and combination therapies. The treatment history of the patients reflects the severity of the epilepsies: the mean number of anticonvulsant drugs previously used was $9.7 \pm 3.8$ (range, 3-16; median, 10). Overall, 2 patients underwent epilepsy surgery; 11 patients had vagal nerve stimulation therapy; and in 13 patients, the ketogenic diet or modified Atkins diet were tried; but in 12 cases, it was discontinued before treatment with PER.

At the time when the PER treatment was initiated, 52 of 58 patients received AEDs. Valproic acid and lamotrigine were the most commonly used concomitant AEDs 
112 Effectiveness and Tolerability of PER in Children and Adolescents Biró et al.

Table 1 Concomitant medication at initiation of PER

\begin{tabular}{|l|l|}
\hline Antiepileptic drug & $n$ \\
\hline Valproic acid & 20 \\
\hline Lamotrigine & 11 \\
\hline Clobazam & 9 \\
\hline Levetiracetam & 8 \\
\hline Ethosuximide & 8 \\
\hline Zonisamide & 6 \\
\hline Topiramat & 6 \\
\hline Phenobarbital & 5 \\
\hline Rufinamide & 5 \\
\hline Oxcarbazepine & 5 \\
\hline Lacosamide & 5 \\
\hline Phenytoin & 3 \\
\hline Bromide & 3 \\
\hline Carbamazepine & 3 \\
\hline Stiripentol & 3 \\
\hline Eslicarbazepine & 3 \\
\hline Felbamate & 2 \\
\hline Clonazepam & 2 \\
\hline Pregabalin & 2 \\
\hline Sultiam & 2 \\
\hline Gabapentin & 1 \\
\hline Retigabin & 1 \\
\hline Tiagabin & \\
\hline Abrevaion & \\
\hline
\end{tabular}

Abbreviation: PER, perampanel.

(-Table 1). Concomitant antiepileptic therapy was continued without modification during the observation period in 41 patients $(71 \%)$.

The titration schedules of PER varied between centers and also interindividually, as currently no recommendations apply to children younger than 12 years. Add-on therapy with PER was usually initiated at $2 \mathrm{mg}$ once daily. Up titration was usually done in $2 \mathrm{mg} / \mathrm{wk}$ or $2 \mathrm{mg} / 2 \mathrm{wk}$ steps, in some cases $2 \mathrm{mg} / \mathrm{d}$ steps. In children younger than 12 years, smaller steps (1 mg) and slower titration rates were applied ( $1 \mathrm{mg} / 2 \mathrm{wk}$ or $2 \mathrm{mg} / 3-4 \mathrm{wk}$ ). The end doses varied according to clinical need.

\section{Results}

The overall response rate ( $\geq 50 \%$ seizure reduction) after the first 3 months of therapy was 31\% (18/58 patients; - Fig. 1). Complete seizure control was achieved in $9 \%$ (5/58 patients), a reduction in seizure frequency of 75 to $99 \%$ in $10 \%(6 / 58$ patients). Notably, children aged 6 years and above tended to show a better response (36\%; $17 / 47$ patients) than younger children ( $9 \% ; 1 / 11$ patients; $p<0.1$; chi-square). Aggravation of seizures (increase of seizure frequency by $\geq 50 \%$ ) occurred in 9\% (5/58 patients; - Fig. 1). - Table 2 displays the subgroup analysis of effectiveness with respect to epilepsy etiology, epilepsy syndromes, and disabling seizure types.

Adverse effects were reported in 28 of 58 patients (48\%). In these patients, a total of 52 adverse effects were registered, of which 43 were considered to be mild or moderate (-Fig. 2 ). The most frequently occurring adverse effects were reduced vigilance or fatigue $(n=16)$ and behavioral changes $(n=14)$. Of latter, aggressiveness $(n=8)$ dominated. Dizziness or gait instability was reported in six cases, changes in appetite were observed in six cases. Appetite loss $(n=3)$ and gain $(n=3)$ were equally represented.

Adverse effects rated as "severe" by the treating physician occurred in seven patients; the total number of severe adverse effects was nine, as two children had two adverse symptoms rated as severe during therapy with PER. In some cases, severe adverse effects seemed to be associated with faster titration rates.

No serious adverse events (according to the FDA classification $^{13}$ ) occurred. The observed adverse effects ceased under dose reduction or discontinuation of PER. Concerning the

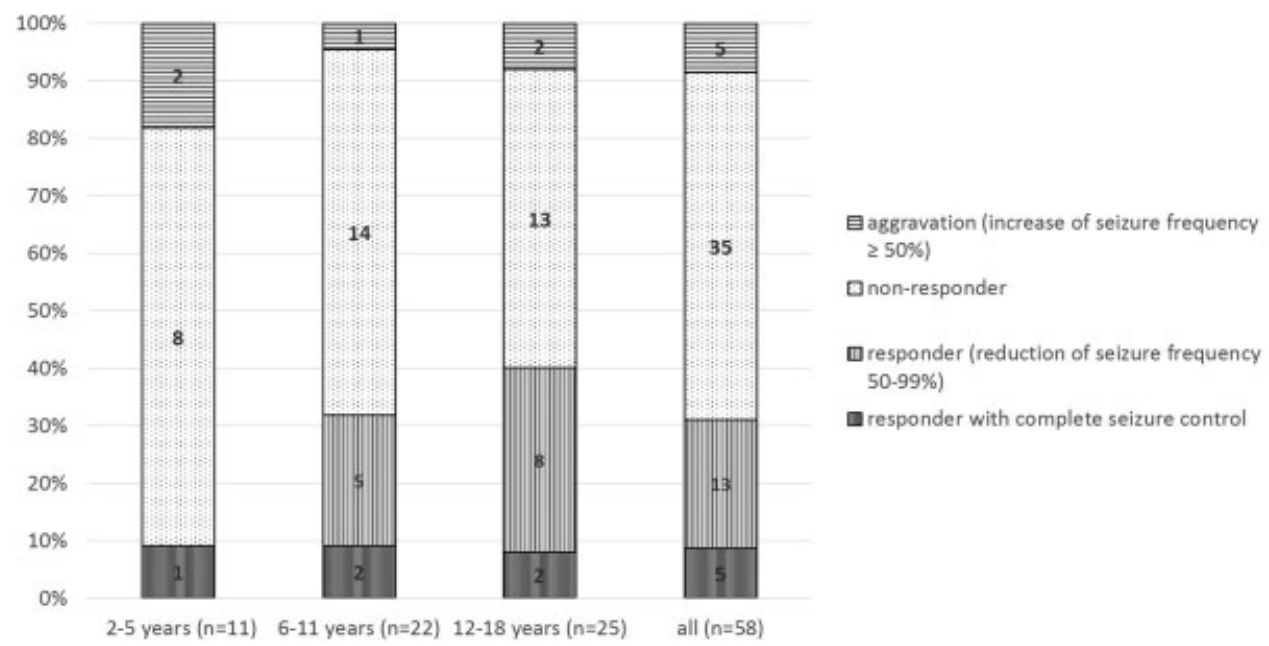

Fig. 1 Effectiveness of perampanel after 3 months of therapy. 
Table 2 Subgroup analysis of effectiveness of PER after 3 months of treatment

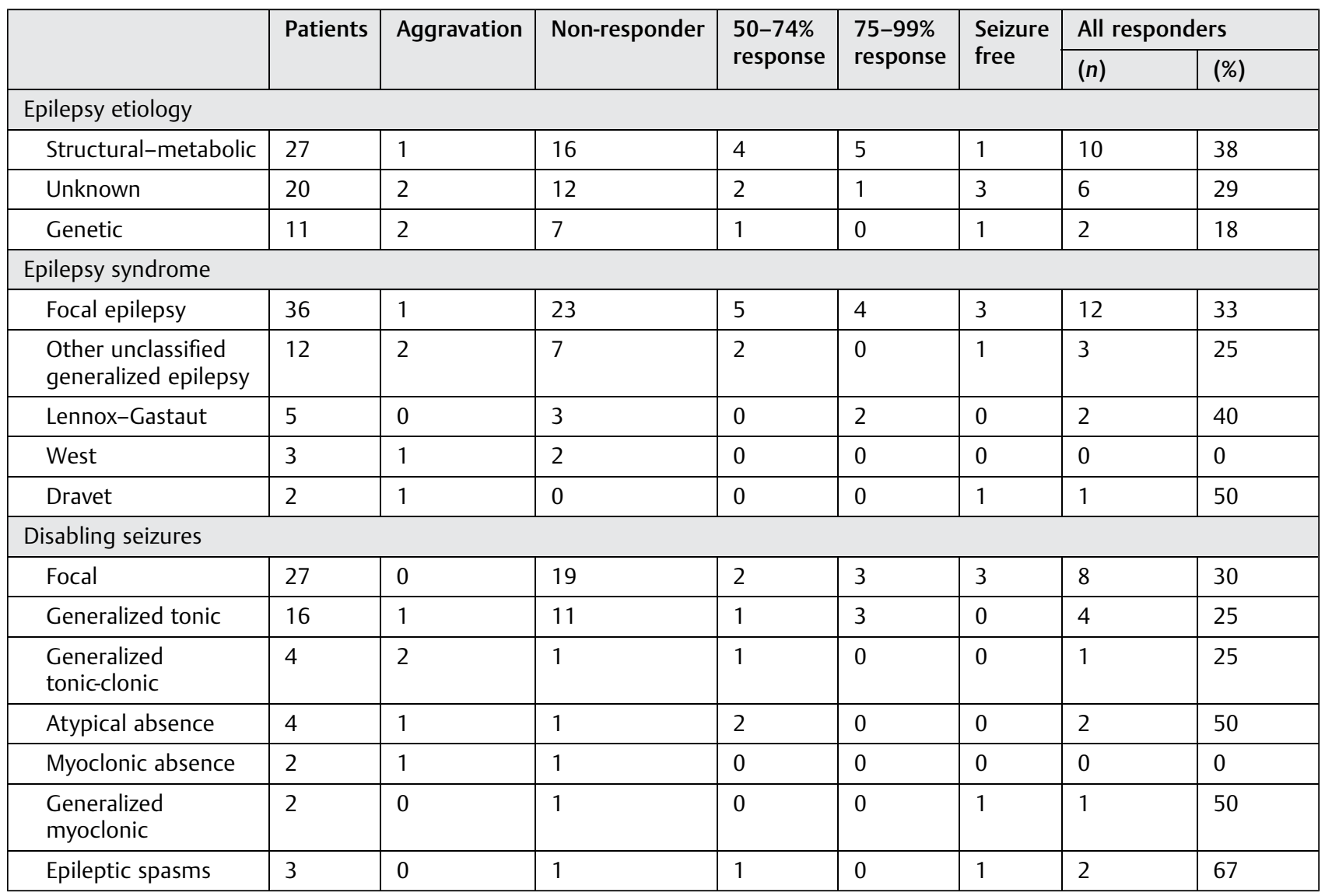

age distribution of adverse effects ( - Fig. 3 ), children younger than 12 years had a more favorable adverse effect profile than the children older than 12 years ( $p=0.037$; chi-square).

\section{Discussion}

PER also seems to be effective in children and adolescents with pharmacorefractory epilepsies: in our cohort of severely refractory epilepsy patients, a significant seizure reduction could be achieved in 18 of 58 cases (31\%). As yet, probably because of the still small cohort, no recommendations can be derived concerning specific epilepsy syndromes or seizure types in which PER is particularly effective.

The high ratio of modification of concomitant therapy (17/58) might appear as a limitation of our results. However, among the 18 patients considered responders, the concomitant medication had only been modified in 5 cases, all of which were discontinuation of 1 or more concomitant AEDs (probably because of the favorable outcome under PER therapy).

Tolerability was acceptable. No serious adverse events occurred, the observed adverse effects ceased under dose reduction or discontinuation of PER. To date, no conclusions can be drawn concerning the long-term effects.

The pharmacokinetic properties of PER (linear concentration-effect and concentration-side effect relationship, steady state only after 2 weeks $)^{6-8}$ suggest that slower titration rates would be advisable to find the smallest possible effective dosage. Probably because of the small cohort, we could not find a direct correlation between titration rates, single dose or twice daily administration, end doses, and the adverse effect rates.

Our results suggest that PER could be better tolerated in the age groups younger than 12 years. Some adverse effects, however, can only be interpreted by patients able to verbalize their disposition, so in this aspect, our data collection may be limited.

Including infants would have been a valuable addition to the collective, but no infants were treated at the involved centers until the initiation of our data collection. This is probably also because of the lack of an oral solution for applying smaller doses. The pediatric investigation plan accepted by the EMA ${ }^{10}$ also includes the development of an oral liquid formulation $(\leq 0.5 \mathrm{mg} / \mathrm{mL}$ ) and studies including infants and newborns, yet completion is only planned by 2021 .

The retrospective character, the uncontrolled study design, the small cohort, and the short observation period are limitations of our study. Because of the lack of prospective and controlled studies in this field, however, we still consider our data worthwhile reporting.

The exchange of such clinical experiences can help to assess the significance of PER in the treatment of children and adolescents with various refractory epilepsies. Furthermore, prospective studies are strongly needed to assess tolerability 


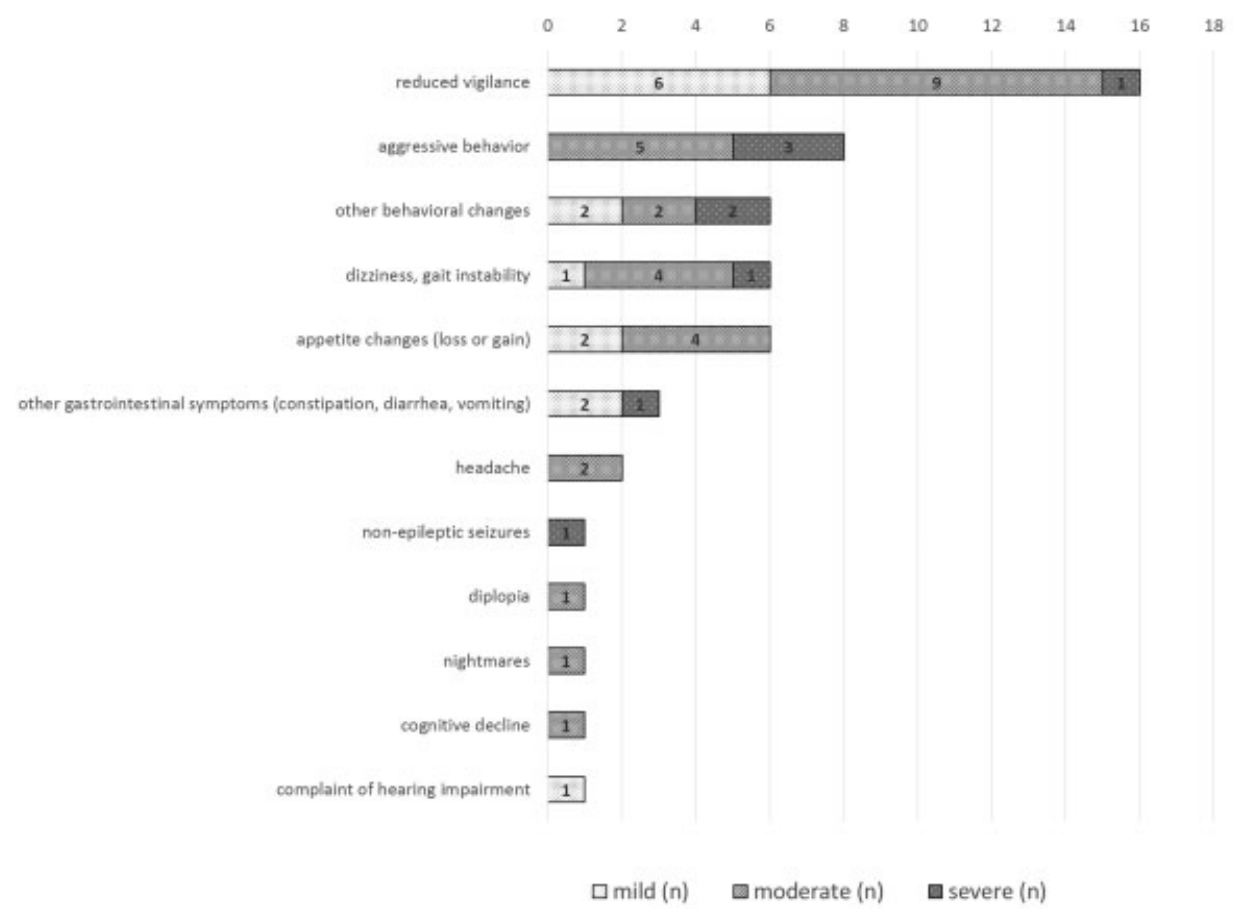

Fig. 2 Adverse effects during the first 3 months of perampanel therapy.

and effectiveness in infancy, in distinct childhood epilepsy syndromes and in rare epilepsy syndromes; and to address long-term tolerability issues, including cognitive evaluation.

\section{Disclosure}

No author has disclosed an association which might affect his or her ability to present and/or interpret data objectively, or might pose a conflict of interest in connection with the article. All coauthors have read and approved the submitted article.
A. Biró has no conflicts of interest to declare. U. Stephani has served on the scientific advisory board for Eisai Inc. T. Bast received honoraria from Eisai Inc. for lectures and as an advisory board member. K. Schlachter has served on the scientific advisory boards for Pfizer, UCB, GSK, Eisai Inc. He has received speaker honoraria from UCB. G. Kurlemann has served on scientific advisory boards for UCB, Novartis, Eisai Inc., Desitin Pharmaceuticals $\mathrm{GmbH}$, and ViroPharma. He has received reimbursement for traveling expenses and/or speaker honoraria from UCB, Eisai Inc., Desitin Pharmaceutical GmbH, ViroPharma, and Novartis. A.

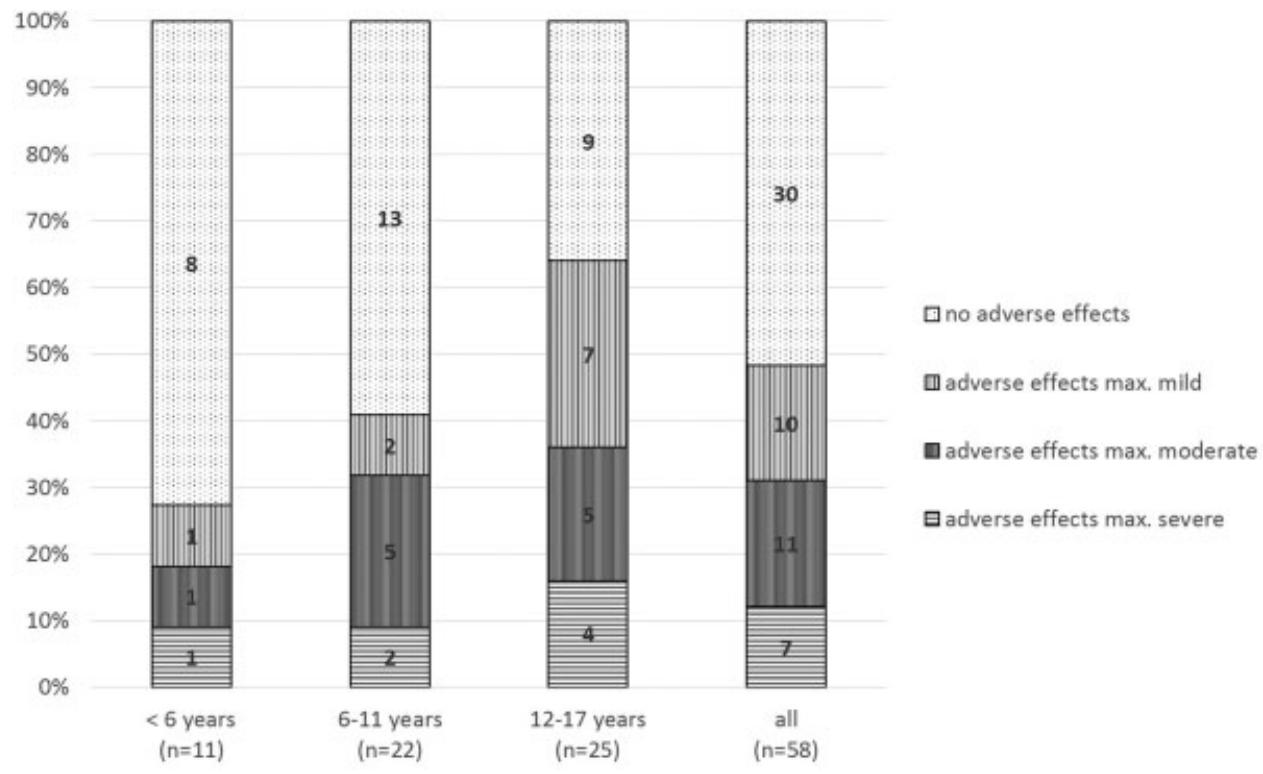

Fig. 3 Age distribution of main adverse effects in the first 3 months of therapy with perampanel. 
Müller has received reimbursement for traveling expenses from Eisai, Desitin, and UCB. M. Staudt has received reimbursement for traveling expenses and/or speaker honoraria from UCB, Desitin Pharmaceutical GmbH, and Medtronic. G. Kluger has served on scientific advisory boards for UCB, Novartis, Eisai Inc., Desitin Pharmaceuticals $\mathrm{GmbH}$, ViroPharma, and Nutricia. He has received reimbursement for traveling expenses and/or speaker honoraria from UCB, Eisai Inc., Desitin Pharmaceutical GmbH, ViroPharma, Novartis, and Nutricia. B. Fiedler, S. Leiz, M. Fleger, M. Nikanorova, M. Wolff, C. Selch, and T. Tarallo have no conflicts of interest to declare.

We confirm that we have read the journal's position on issues involving ethical publication and affirm that this report is consistent with those guidelines.

\section{References}

1 Hanada T, Hashizume Y, Tokuhara N, et al. Perampanel: a novel, orally active, noncompetitive AMPA-receptor antagonist that reduces seizure activity in rodent models of epilepsy. Epilepsia 2011; 52(7):1331-1340

2 Ceolin L, Bortolotto ZA, Bannister N, Collingridge GL, Lodge D, Volianskis A. A novel anti-epileptic agent, perampanel, selectively inhibits AMPA receptor-mediated synaptic transmission in the hippocampus. Neurochem Int 2012;61(4):517-522

3 French JA, Krauss GL, Biton V, et al. Adjunctive perampanel for refractory partial-onset seizures: randomized phase III study 304 . Neurology 2012;79(6):589-596

4 French JA, Krauss GL, Steinhoff BJ, et al. Evaluation of adjunctive perampanel in patients with refractory partial-onset seizures: results of randomized global phase III study 305. Epilepsia 2013;54(1):117-125

5 Krauss GL, Serratosa JM, Villanueva V, et al. Randomized phase III study 306: adjunctive perampanel for refractory partial-onset seizures. Neurology 2012;78(18):1408-1415

6 European Medicines Agency. Fycompa: EPAR - Product Information. 2012. Available at http://www.ema.europa.eu/docs/en_GB/ document_library/EPAR_-_Product_Information/human/002434/ WC500130815.pdf. Accessed August 31, 2014

7 Food and Drug Administration. Fycompa - NDA 202834, FDA Approved Labeling Text. 2012 Okt. Available at http://www. accessdata.fda.gov/drugsatfda_docs/label/2012/202834lbl.pdf. Accessed August 31, 2014

8 Gidal BE, Ferry J, Majid O, Hussein Z. Concentration-effect relationships with perampanel in patients with pharmacoresistant partial-onset seizures. Epilepsia 2013;54(8):1490-1497

9 Steinhoff BJ, Bacher M, Bast T, et al. First clinical experiences with perampanel-the Kork experience in 74 patients. Epilepsia 2014; 55(Suppl 1):16-18

10 European Medicines Agency decision P/0160/2014 of 11 June 2014 on the acceptance of a modification of an agreed paediatric investigation plan for perampanel (Fycompa), (EMEA-000467PIP01-08-M05) in accordance with Regulation (EC) No 1901/ 2006 of the European Parliament and of the Council. 2014 Jun. Available at: http://www.ema.europa.eu/docs/en_GB/document_ library/PIP_decision/WC500169857.pdf. Accessed August 31, 2014

11 Amann JP, Glauser T, Chiron C. Developing antiepileptic drugs in children: balancing protection and access. Handb Clin Neurol 2013;111:741-746

12 Berg AT, Berkovic SF, Brodie MJ, et al. Revised terminology and concepts for organization of seizures and epilepsies: report of the ILAE Commission on Classification and Terminology, 2005-2009. Epilepsia 2010;51(4):676-685

13 Food and Drug Administration. CFR - Code of Federal Regulations Title 21, Volume 5; 21CFR312.32, 2014 
116 Effectiveness and Tolerability of PER in Children and Adolescents Biró et al.

Supplementary Table 1 Participating centers

\begin{tabular}{|l|l|}
\hline & Number of patients \\
\hline $\begin{array}{l}\text { Department of Neuropediatrics, University Medical Center Schleswig-Holstein, } \\
\text { Christian-Albrechts University, Kiel, Germany }\end{array}$ & 16 \\
\hline Epilepsy Center Kork, Kehl, Germany & 16 \\
\hline $\begin{array}{l}\text { Department of Pediatric Neurology and Neurological Rehabilitation, Epilepsy Center } \\
\text { for Children and Adolescents, Schün Klinik Vogtareuth, Germany }\end{array}$ & 8 \\
\hline Department of Pediatrics, LKH Bregenz, Bregenz, Austria & 5 \\
\hline Department of Neuropediatrics, University Children's Hospital Münster, Germany & 4 \\
\hline Department of Pediatrics, Dritter Orden Clinic, Munich, Germany & 3 \\
\hline Danish Epilepsy Centre, Dianalund, Denmark & 3 \\
\hline Department of Pediatric Neurology, University Children's Hospital Tübingen, Germany & 3 \\
\hline
\end{tabular}

